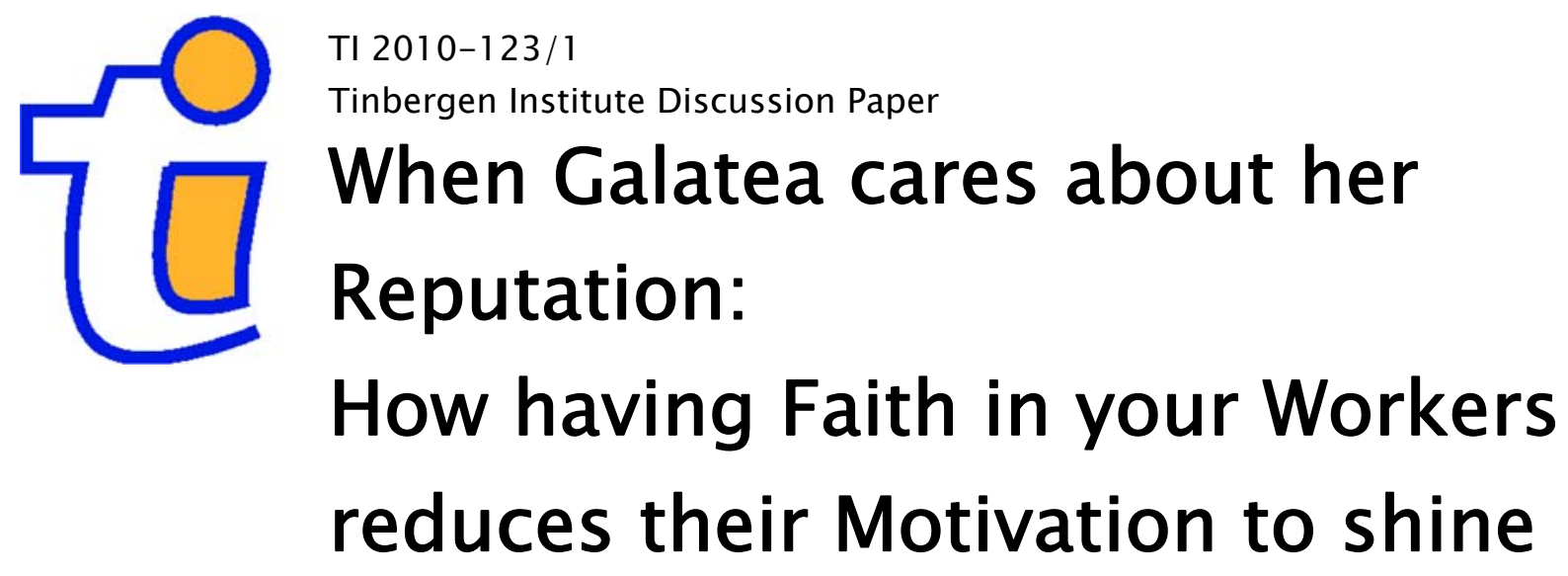

Jurjen J.A. Kamphorst

Otto H. Swank*

Erasmus School of Economics, Erasmus University Rotterdam.

* Tinbergen Institute. 


\section{Tinbergen Institute}

The Tinbergen Institute is the institute for economic research of the Erasmus Universiteit Rotterdam, Universiteit van Amsterdam, and Vrije Universiteit Amsterdam.

Tinbergen Institute Amsterdam

Roetersstraat 31

1018 WB Amsterdam

The Netherlands

Tel.: +31(0)205513500

Fax: $+31(0) 205513555$

Tinbergen Institute Rotterdam

Burg. Oudlaan 50

3062 PA Rotterdam

The Netherlands

Tel.: + $31(0) 104088900$

Fax: $+31(0) 104089031$

Most TI discussion papers can be downloaded at http://www.tinbergen.nl. 


\title{
When Galatea cares about her reputation: how having faith in your workers reduces their motivation to shine
}

\author{
Jurjen J.A. Kamphorst ${ }^{1}$ \\ Erasmus School of Economics \\ kamphorst@ese.eur.nl
}

\author{
Otto H.Swank \\ Erasmus School of Economics \\ and Tinbergen Institute \\ swank @ese.eur.nl
}

December 6, 2010

\begin{abstract}
We develop a theory of leadership that focuses on the role managers play in motivating employees through their attitude towards employees. We model a manager's attitude as her perception about employees' abilities of successfully completing challenging tasks. We show that a positive attitude motivates employees who are driven by monetary rewards. A negative attitude may motivate employees who are driven by concerns about their reputation for being able. When employees are driven by monetary rewards and care about their reputations, an increase in the reward for successfully completing challenging tasks may lead employees to shy away from these tasks.
\end{abstract}

Keywords: reputation, worker motivation, task choice, principle-agent, Giffen goods.

JEL: D82, M59.

\footnotetext{
${ }^{1}$ We thank Josse Delfgaauw, Robert Dur, Chaim Fershtman, Ewa Mendys-Kamphorst, Sander Renes and participants of the ESE Brown Bag seminar for their helpful comments.
} 


\section{Introduction}

A famous myth tells about Pygmalion, a prince of Cyprus, who carved a sculpture of a woman out of ivory. Pygmalion felt in love with his statue. Through his will and the assistance of Venus, the statue changed to a real woman, Galatea. In the psychological literature, the myth of Pygmalion is frequently used as a metaphor for two well-documented phenomena in organizations. First, high expectations of a manager about a subordinate induce the manager to pay special attention to the subordinate resulting in higher performance. This is the Pygmalion effect. The emphasis is on the manager working harder. Second, high expectations conveyed directly to the subordinate also result in higher performance. This is called the Galatea effect. The emphasis is on the subordinate working harder. Both effects are examples of self fulfilling prophecies. The psychological literature provides a lot of evidence for the relevance of both effects [see, for example, Eden (1990), Eden and Kinnar (1991), Eden (1992), Oz and Eden (1994), McNatt (2000), Shapiro et al. (2007), Bezuijen et al. (2009), and Kierein and Gold (2000) for a meta-analysis]. Eden (1992), for instance, conducted several field experiments on men who had been selected into a combat command course. The experiments showed that manipulation of both the instructors' expectations and the trainees' expectations has large effects on trainees' performances.

The Galatea effect is mediated by subordinates' raised expectations of themselves. The way people see themselves drives many of their decisions [see Bandura (1986) on the relationship between self-efficacy and behavior]. People who think highly of their abilities work harder, are more likely to choose challenging tasks or are less likely to give up difficult tasks than people who think poorly of themselves. In a business setting, the relationship between people's self perception on the one hand and their motivation on the other creates a role for managers. Conveying particular expectations to subordinates becomes a means of motivating them.

A good manager boosts an employee's sense of self-efficacy in order to induce greater effort and better performance. At least, this is the view that emerges from the literature on the Pygmalion and Galatea effect. There might be other forces at work, however. For instance, employees should not be too confident about their abilities. Overconfident employees may choose too ambitious tasks resulting in poor 
performance. Moreover, employees may have weaker incentives to impress their supervisor if he already has a very favourable view of their abilities. A supervisor having low expectations may challenge employees. These examples suggest that there might be limits to the extent to which supervisors should boost employees senses of self-efficacy. The main objective of the present paper is to shed light on the pros and cons of boosting employees' perceptions of their abilities.

We develop a model in which a manager's attitude towards an employee is defined by the probability she believes this employee can successfully perform a challenging task. A manager with a more positive attitude considers it more likely that an arbitrary employee can perform a challenging task than a manager with a more negative attitude. In our model, the employee is driven by (monetary) rewards for successfully completing tasks. Moreover, the employee is concerned about how the manager perceives his ability. One reason for this motive is that the manager's perception may affect the employee's future career perspectives. Alternatively, the employee may derive direct utility from being valued highly. The employee makes two decisions. He either chooses to perform a routine task or a challenging task. Next, if he has opted for the challenging task, he chooses an effort level. The probability that the employee successfully completes a challenging task depends on his ability and the effort level. The manager is a passive player. She forms a view of employees' abilities. Furthermore, through her attitude towards employees, she affects employees' behavior.

A manager's attitude affects the employee's behavior through two channels. First, by learning the manager's attitude, the employee updates his belief about his own abilities. For example, an employee who enters a company with a belief that with a high probability he is able to perform challenging tasks adjusts his belief downwards when he learns that the manager beliefs that hardly any employee can perform challenging tasks. Of course, this channel requires that the employee attributes a positive probability to the event that the manager's vision contains relevant information. Second, a manager's attitude on employees influences the way she updates her beliefs about employees. The reason is simply that posterior beliefs depend on outcomes and prior beliefs. This second channel is important when workers want to come across as able.

We derive five main results. First, we derive the Galatea effect. Employees who 
have a positive self-image tend to perform more challenging tasks and put more effort in them than employees with a negative self-image. This result is neither new nor surprising. It stems from the following features of the model: (i) more confident employees are more likely to complete challenging tasks; and (ii) effort levels depend positively on the probability of being able. The implication of our first result is that managers with a positive attitude induce employees to choose challenging tasks and to put more effort in those tasks. In our model, given task choice, a higher level effort is always good from the manager's point of view. A stronger inclination to choose the challenging is task is not always good. Whether this is good or bad depends on whether the employee can actually handle the challenging task.

Second, an extreme attitude of the manager may distort self-selection. Even though employees sometimes have an incorrect view of their own abilities, their views contain some information. For this reason, a manager may want to rely on self-selection: allowing employees to choose the tasks they want to perform. An employee who has a favorable view of his own abilities may then choose challenging tasks, while an employee with a more negative view may choose routine tasks. An extreme view by the manager may distort self-selection in case a negative view discourages high ability types to perform challenging tasks, or in case a positive view encourages low ability types to perform challenging tasks. In some organizations, managers try to break employees' self images to prevent them from taking unwanted initiatives. In other organizations, managers may try to boost employees' self images to stimulate risk-taking.

Third, a manager's belief has important consequences for employees' incentives to prove themselves. In the presence of a manager who holds a positive belief about employees, a low ability type has little to gain in terms of reputation and much to lose by performing challenging tasks. In case of a more sceptical manager, by contrast, a high ability type has much to win by performing a challenging task. Indeed, we show that a manager holding a negative belief may encourage high ability employees to perform challenging tasks and to put much effort in them.

Fourth, raising high expectations about employees' abilities is good for performance when employees are motivated by monetary rewards. Then, higher selfefficacy always encourages an employee to expend effort (the first result above). However, in an environment where employees are predominantly motivated by ca- 
reer concerns, raising high expectations can be counterproductive. As discussed in the third result above, employees' incentives to work hard to come across as able may be weak in case managers already have a positive view. Thus, which managers in terms of their attitude towards employees' abilities motivate the employee the most hinges on the specific environment in which a manager operates.

Finally, we show that an increase in the reward for completing the challenging task may induce workers to shy away from this task. To understand this somewhat counter-intuitive result, it is important to recall an important feature of models with agents having reputational concerns. In equilibrium, expectations of reputations cannot be influenced. In our model, this means that when the worker makes his task decision, the expected reputation belonging to each task is fixed. In a separating equilibrium, the challenging task yields a better reputation as a choice for the challenging task suggest a high ability. An employee anticipates that the choice for the challenging task will lead him to expend effort with a view of impressing the manager. In equilibrium, this effort does not affect the employees' reputation. From the worker's point of view the effort is to a large extent a waste. Now consider an increase in the monetary reward. The direct effect of a higher reward for completing a challenging task is that the employee expends more effort. This, in turn, implies that failing on the challenging task provides more information about a worker's ability. Failing becomes a clearer signal of disability. This motivates the worker to put even more effort in the task. This extra effort is a cost of choosing the challenging task. By choosing the routine task instead of the challenging task, the employee steps out of the rat race.

All in all our paper contributes to our understanding of how a personal characteristic of managers, here their views of employees' abilities, influence the behavior of employees and the performance of the company. Our analysis shows that a manager's view of employees' abilities may be important even if it does not contain any information. Furthermore, our analysis shows under which conditions a more positive view motivates, and under which conditions a more negative view motivates.

This papers builds on three recent strands in the literature. First, our paper builds on recent work that emphasizes that employees try to infer information about their abilities from how they are treated by their managers [see, for example, Benabou and Tirole (2003), Ishida (2006), and Swank and Visser (2007)]. The point of 
departure in these studies is that employees have imperfect knowledge about their own abilities. In the social psychological literature, the evidence for this phenomenon abounds [Sedikes and Strube (1995), Klar et al. (1996), Baumeister (1998), Kruger (1999), and Ackerman et al. (2002]. Imperfect knowledge about oneself can lead to errors. People may perform tasks they cannot handle or abstain from performing tasks they could have handled. Indeed, Ehrlinger and Dunning (2003) find that women disproportionately avoid careers in science because they underestimate their reasoning abilities. In Benabou and Tirole (2003) and Swank and Visser (2007), managers give challenging tasks to subordinates to induce them to exert much effort. In this strand of literature, the emphasis is on how manager's actions influence employees self-efficacy, and in turn performance. These studies thus model the Pygmalion effect. In the present paper, employees themselves choose tasks. $^{2}$ Employees are not influenced by a manager's direct choices, but by the manager's general view on employees' abilities. Our paper deals with the Galatea effect. Our finding that providing rewards may have adverse effects is not new. In Benabou and Tirole (2003) a monetary reward may crowd out intrinsic motivation because a monetary reward may contain information about the task. For example, a high reward may signal that the task is boring. In Benabou and Tirole (2006), monetary rewards may be counterproductive because they reduce the reputational benefits of doing good. As far as we know, our explanation for the possible adverse effect of providing monetary rewards is new. As explained above, it hinges on a spillover between monetary rewards and reputational concerns. Monetary rewards may induce employees to exert so much effort that employees prefer to expend no effort at all.

Second, our paper is related to studies exploring the effects of heterogenous prior beliefs. In many economic models, agents may disagree with each other because they have private information. In those models, agents usually have common priors. Recently, some authors have dropped the assumption of common priors. For example, Van den Steen (2005) models managerial vision as the belief a manager holds about

\footnotetext{
${ }^{2}$ Köszegi (2006) also considers an agent who can select his own task. This agent cares about his self-esteem. As a result, he may choose to avoid challenging, profitable tasks when such tasks may reveal information which diminishes his self-esteem beyond some cut-off point. The results by Köszegi (2006) depend on the existence of this cut-off point, whereas we will not have any such cut-off value in our model.
} 
the right course of action. ${ }^{3}$ People may differ in their beliefs on the right course of action. A strong belief by a manager may attract employees holding similar beliefs. In our model, we think of the employee as a junior entering a new environment with some prior about his own abilities. In this new environment, the junior may learn that other people, such as his manager, hold other priors. We assume (through some Bayesian updating) that this new experience has some effect on the junior's prior. However, even if we had assumed that the junior does not learn from the priors held by his manager, heterogenous priors would have important implications for the behavior of the employee. The reason is that posterior beliefs depend partially on prior beliefs. As a result, when trying to impress the manager, employees take the prior of the manager into account.

Third, this paper contributes to the literature on the various aspects of leadership. By far and large, the main role of managers in economic models is the provision of pecuniary incentives. The number of studies paying attention to other roles of managers is relatively limited. Some examples are worth mentioning. As discussed before, Van den Steen (2005) shows how a manager's view on the right course of action may attract employees sharing this vision (an earlier paper on vision is by Rotemberg and Saloner, 1993). Hermalin (1998) shows how leading by example may motivate employees. Benabou and Tirole (2003), finally, show how delegation of tasks may encourage employees. The present paper is most closely related to Van den Steen (2005) in that it is a personal characteristic of the manager that influences the behavior of employees.

This paper is organized as follows. We present the model in Section 2. Next, we present the analysis in Sections 3-5. Section 6 concludes.

\section{The Model}

\section{The players and their actions}

There are two players in the game, the manager and a worker. The worker must perform a task $X$. There are two tasks, a routine one $(T R)$ and a challenging one

\footnotetext{
${ }^{3}$ Other examples of studies using heterogenous prior beliefs are Banerjee and Somanathan (2001), Dixit and Weibull (2007) and Che and Kartik (2008).
} 
$(T C): X \in\{T R, T C\}$. Workers can be distinguished on the basis of their abilities $t=\{A, N A\}$. An able worker $(t=A)$ can successfully complete a challenging task if he puts sufficient effort $(e)$ in it. A less able worker $(t=N A)$ can never complete a challenging task. We denote by $Z \in\{S, F\}$ the outcome of a task with $Z=S$ meaning that a task is successfully completed, and $Z=F$ meaning that the worker failed to complete the task. We assume that the probability with which an able worker succeeds at the challenging task is increasing in his effort. To model this in a simple way we assume that there is an unknown difficulty level $y$ which is drawn from a known, uniform distribution on the interval $[0,1]$. The worker succeeds at $T C$ if his effort matches or exceeds $y$ and he is able. Otherwise he fails. For $X=T C$, $Z=S$ requires that $t=A$ and $e \geq y$. If $t=N A$ or $e<y$, then $Z=F$. Completing a routine task does not require effort nor a specific type. Therefore, for $X=T R$, $Z=S$.

The manager plays two roles. First, she has an expectation regarding the worker's ability. This expectation is defined as the probability with which the manager believes the worker is able, $\alpha_{M}$. Second, at the end of the game the manager updates her belief about the worker's ability on the basis of the worker's behavior

and task performance. As we will discuss below, to model the Galatea effect, we assume that the worker knows $\alpha_{M}$. In fact, investigating the Galatea effect amounts to investigating the effect of $\alpha_{M}$ on the worker's behavior.

\section{The information structure and the timing}

Our model tries to capture a couple of features. First, a new worker enters an organization with a notion about his ability. Second, he learns the manager's expectation regarding him $\left(\alpha_{M}\right)$. This expectation may be based on many sources, such as (but not limited to) the manager's knowledge of the job, his experience with other workers, his impression of the worker, or even prejudices. The important assumption is that the manager's opinion may contain relevant information, and that this may change the worker's perception of his ability. Third, when getting acquainted with work, the worker develops a sense of whether he has a talent for the job. In line with these features, we make the following assumptions.

At the beginning of the game nature draws $y$ and $t$. Neither $y$ nor $t$ is observed at any time by the worker and the manager. We denote by $\alpha_{t}$ the true probability 
that $t=A$. This probability is not common knowledge. Rather, we assume that initially the worker believes that the probability that he is able is $\alpha_{W}$, while the manager, knowing $\alpha_{W}$, believes that this probability is $\alpha_{M}$. The worker knows $\alpha_{M}$. To model the worker's initial learning as simple as possible, we assume that the worker attributes a probability $\rho_{M}$ that $\alpha_{M}$ is the true probability that $t=A$, and a probability $1-\rho_{M}$ that $\alpha_{W}$ is the true probability that he is able. ${ }^{4}$ In this way, we model the Galatea effect. When choosing a task and effort level the worker believes that the probability with which he is able equals $\alpha=\rho_{M} \alpha_{M}+\left(1-\rho_{M}\right) \alpha_{W}$. To minimize burdensome notation, we assume that the worker's initial belief about the probability that $t=A\left(\alpha_{W}\right)$ does not affect the manager's belief that $t=A$. To model that the worker develops a sense of whether he has a talent for the job, we assume that the worker receives a private signal about his ability: $s \in\{A, N A\}$, with $\operatorname{Pr}(s=t)=\rho$, where $\frac{1}{2}<\rho<1$, and $\operatorname{Pr}(s \neq t)=1-\rho$. Both the manager and the worker know that $s$ contains information about the worker's ability. Throughout, we refer to a worker who has received $s=A(s=N A)$ as an $A$ worker ( $N A$ worker).

Having processed the information about his ability, the worker chooses a task $X$ next. In case $X=T C$, the worker also chooses $e$. After the worker has expended effort (provided $X=T C$ ), it becomes clear whether or not the challenging task was completed successfully. At the end of the game, the manager forms a belief about the worker's ability. The manager observes $X$. Moreover, if $X=T C$, she observes $Z$. However, the manager does not observe $t, y, e$ and $s$. The distribution of $y$ is common knowledge. The manager updates his beliefs about the probability that the worker is able using Bayes' rule. We denote by $\hat{\alpha}(\cdot)$ this posterior probability.

\section{Payoffs, strategies, and equilibria}

Successfully completing a task yields a reward to the worker, $W_{X}$. Furthermore, the worker is concerned with how the manager views his ability. Finally, the worker is effort averse. Specifically, we assume that the worker's preferences are described by

\footnotetext{
${ }^{4} \mathrm{~A}$ motivation for this structure is that there are two types of managers. Managers who have learnt from their experience and have developed a correct attitude towards workers, and managers who have not learnt anything and whose attitudes do not contain any information. The probability that a manager is of the first type equals $\rho_{M}$.
} 
the following three equations:

$$
\begin{aligned}
U(T C, e, S) & =W_{T C}+\lambda \hat{\alpha}(T C, S)-\frac{1}{2} e^{2} \\
U(T C, e, F) & =\lambda \hat{\alpha}(T C, F)-\frac{1}{2} e^{2} \\
U(T R) & =W_{T R}+\lambda \hat{\alpha}(T R)
\end{aligned}
$$

where $\lambda$ denotes the weight the worker attributes to his reputation. Equations (1) and (2) denote the worker's payoff if he chooses a challenging task and succeeds respectively fails to complete it. Equation (3) gives the worker's payoff, if he performs the routine task.

The strategy of the worker consists of two parts. First, he chooses a task $\tau:(s) \rightarrow\{T R, T C\}$. Second, the worker chooses an effort level $e:(s, X) \rightarrow[0, \infty)$. The manager uses a belief updating function $\hat{\alpha}:(X, Z) \rightarrow[0,1]$. We apply the concept of Perfect Bayesian equilibrium to solve the game. Our focus is on equilibria in pure strategies. It is well-known that in the present kind of games, two types of equilibria in pure strategies exist: separating equilibria and pooling equilibria. In a separating equilibrium, the manager infers the worker's type (note that in the present model, $s$ is the worker's type) from $X$. In a pooling equilibrium, she does not. In the present model, $A$ workers are better equipped to perform the challenging task than $N A$ workers. For this reason, if a separating equilibrium exists it is one in which $A$ workers perform $T C$ and $N A$ workers $T R$. We denote by $\sigma(X)$ the manager's belief about the probability that the worker is of type $A$. In a separating equilibrium, $\sigma(T C)=1$ and $\sigma(T R)=0$. In a pooling equilibrium where both types of workers choose $T R$, the manager does not learn anything about the worker's ability $\sigma(T R)=\alpha_{M}$. In a pooling equilibrium where both types of workers choose $T C$, posterior beliefs depend on task performance. We assume that the parameters of the model are such that in any equilibrium $e(s, X)<1$ for all $s$ and $X$. The implication of this assumption is that regardless of the worker's ability, in equilibrium there exists a strictly positive probability that the worker fails to complete the challenging task. As mentioned earlier, the manager is a passive player. Her influence on the worker runs through $\alpha_{M}$. Below we treat the model as if the manager wants workers to expend much effort. Moreover, the manager may want workers to self-select: $A$ workers to perform $T C$, and $N A$ workers to perform 
$T R$.

The analysis proceeds as follows. In Section 3 we consider the case where reputational concerns play no role. Instead, the worker is incentivized through performance wage. In Section 4 we consider the opposite case, namely where there is no performance wage and the worker is stimulated (or not) through his reputational concerns only. Finally, in Section 5 we discuss how performance wage and reputational concerns interact.

\section{No reputational concerns $(\lambda=0)$}

In this section, we assume that workers are not concerned about their reputation $(\lambda=0)$ and $W_{T C}>W_{T R}>0$. The analysis consists of three steps. First, we determine how much effort the worker puts in a challenging task. Next, we identify the conditions under which a separating equilibrium and pooling equilibria exist. Finally, we examine how the worker's perception of his ability in general $(\alpha)$, and the manager's perception of the worker's ability in particular $\left(\alpha_{M}\right)$, affect the worker's effort and task choices.

First, consider the worker's effort decision. As the completion of a routine task does not require any effort, suppose that $X=T C$. Then a worker's expected utility equals

$$
\begin{aligned}
E[U(T C, e, Z \mid s)] & =\operatorname{Pr}(t=A \mid s) \operatorname{Pr}(y \leq e) W_{T C}-\frac{1}{2} e^{2} \\
& =\operatorname{Pr}(t=A \mid s) e W_{T C}-\frac{1}{2} e^{2}
\end{aligned}
$$

where $\operatorname{Pr}(t=A \mid s)$ can be interpreted as the worker's self image after observing his signal $s$. Maximizing (4) with respect to $e$ yields

$$
e(s)=\operatorname{Pr}(t=A \mid s) W_{T C}
$$


with

$$
\begin{aligned}
& \operatorname{Pr}(t=A \mid s=A)=\frac{\alpha \rho}{\alpha \rho+(1-\alpha)(1-\rho)}> \\
& \operatorname{Pr}(t=A \mid s=N A)=\frac{\alpha(1-\rho)}{\alpha(1-\rho)+(1-\alpha) \rho}
\end{aligned}
$$

Equation (5) shows that a worker's effort depends positively on his perception of his ability. Together (5) and (6) imply that $A$ workers put more effort in TC than $N A$ workers. As $\alpha_{M}$ affects $\alpha$ positively [recall that $\alpha=\rho_{M} \alpha_{M}+\left(1-\rho_{M}\right) \alpha_{W}$ ], and in turn $\alpha$ affects $\operatorname{Pr}(t=A \mid s)$ positively, the more positive the manager's view of worker's ability is, the more effort that worker expends. This is one form of the Galatea effect: higher expectations lead to higher expected performance.

Let us now consider the worker's task choice. In a separating equilibrium, an $A$ worker chooses $X=T C$, and an $N A$ worker $X=T R$. Neither type should have an incentive to deviate. When choosing a task, a worker anticipates how much effort he will put in a challenging task. Using (5) and (6), one can show that a separating equilibrium exists if the following two conditions are satisfied

$$
\begin{aligned}
E\left[U\left(T C, e_{S E}^{*}(A), Z\right) \mid s=A\right] & \geq U(T R) \\
\frac{1}{2}\left(\frac{\alpha \rho}{\alpha \rho+(1-\alpha)(1-\rho)}\right)^{2}\left(W_{T C}\right)^{2} & \geq W_{T R}
\end{aligned}
$$

and

$$
\begin{aligned}
E\left[U\left(T C, e_{S E}^{*}(N A), Z\right) \mid s=N A\right] & <U(T R) \\
\frac{1}{2}\left(\frac{\alpha(1-\rho)}{\alpha(1-\rho)+(1-\alpha) \rho}\right)^{2}\left(W_{T C}\right)^{2} & <W_{T R}
\end{aligned}
$$

where $e_{S E}^{*}(s)$ is the equilibrium effort by an $s$ type in a separating equilibrium [see (5)]. As $\rho>\frac{1}{2}$, the term in large brackets in (7) is higher than the term in large brackets in (8). It follows that for some ranges of $W_{T C}$ and $W_{T R}$ a separating equilibrium exists. Equations (7) and (8) are also the conditions for the pooling equilibria. If (7) is violated, a pooling equilibrium exists where both types of workers choose the routine task. If, by contrast, (8) is violated, a pooling equilibrium exists in which both types perform the challenging task.

For completeness, we should give the posterior probabilities that the worker is 
smart. In the present model, these probabilities do not influence the payoffs of the worker $(\lambda=0)$, and thus they do not affect the worker's behavior. For this reason, we postpone a discussion about the posteriors to the next section where the focus of the analysis is on reputational concerns.

There remains the question how changes in the parameters of the model affect the incentive compatibility constraints (7) and (8). Clearly, the more informative the worker's signal $s$ is (higher $\rho$ ), the wider is the range of parameters for which a separating equilibrium exists. Moreover, through $\alpha$, a rise in $\alpha_{M}$ increases the left-hand side of both (7) and (8). As a result, a more positive view of the worker's ability held by the manager strengthens both types of workers' incentives to perform the challenging task. This can be interpreted as a second form of the Galatea effect. Higher expectations induce workers to choose more challenging tasks. Note that the Galatea effect on effort is always good from the manager's perspective. Given that a worker performs a challenging task, the manager wants him to work hard on it. The Galatea effect on task choice may, however, make the manager worse off. For instance, it may induce $(N A)$ workers to perform challenging tasks, while the manager wants them to perform routine tasks.

\section{Only reputational concerns $\left(W_{T C}=W_{T R}=0\right)$}

We now turn to the case where workers are not motivated by means of wages $\left(W_{T C}=W_{T R}=0\right)$. Rather workers are driven by concerns about their reputation, so $\lambda>0$. We identify the conditions under which a separating equilibrium exists in which an $A$ worker chooses $T C$ and an $N A$ worker $T R$. We proceed as follows. Assuming a separating equilibrium, we derive the posterior probabilities of the worker's abilities. Next, we determine the effort level a worker puts in a challenging task. Subsequently, we derive the incentive compatibility constraints for the separating equilibrium. Finally, we use numerical simulations to show the existence of a separating equilibrium.

When updating her beliefs about the worker's ability, the manager observes (i) the task $X$, and (ii) if $X=T C$, performance $(Z)$. Moreover, she anticipates some effort from an $A$ worker doing the challenging task, $e_{S E}^{a n t}(A)$. From $Z=S$, the manager infers that the worker is able, as only able workers can complete a 
challenging task. We denote this by $\hat{\alpha}(T C, S)=1$. If $Z=F$, the manager infers that the worker has received a positive signal. Bayes' rule implies

$$
\hat{\alpha}\left(T C, F ; e_{S E}^{a n t}(A)\right)=\frac{\alpha_{M} \rho\left[1-e_{S E}^{a n t}(A)\right]}{\alpha_{M} \rho\left[1-e_{S E}^{a n t}(A)\right]+\left(1-\alpha_{M}\right)(1-\rho)}
$$

Two features of (9) are worth emphasizing. First, the more effort is anticipated, the stronger failing on $T C$ is a signal of incompetence: $\hat{\alpha}\left(T C, F ; e_{S E}^{a n t}(A)\right)$ depends negatively on $e_{S E}^{a n t}(A)$. Second, the posterior depends on the manager's prior belief about the worker's ability, $\alpha_{M}$. A worker failing on $T C$ does not severely damage his reputation if $\alpha_{M}$ is high.

If $X=T R$, then Bayes' rule implies

$$
\hat{\alpha}(T R)=\frac{\alpha_{M}(1-\rho)}{\alpha_{M}(1-\rho)+\left(1-\alpha_{M}\right) \rho}
$$

Note that the reputation of a worker performing $T R$ also depends positively on $\alpha_{M}$. As a consequence, a manager with a positive view of workers (high $\alpha_{M}$ ) concludes from $X=T R$ that it is likely that the worker has received a wrong signal. In contrast, if $\alpha_{M}$ is low, $X=T R$ confirms the manager's initial view.

Now consider the worker's effort choice. Suppose $X=T C$. When choosing effort, the worker's expected utility equals

$$
\begin{aligned}
E[U(T C, e, Z \mid s)]= & \operatorname{Pr}(t=A \mid s) \lambda\left[\operatorname{Pr}(y \leq e) \hat{\alpha}(T C, S)+\operatorname{Pr}(y>e) \hat{\alpha}\left(T C, F ; e_{S E}^{\text {ant }}(A)\right)\right] \\
& +\operatorname{Pr}(t=N A \mid s) \lambda \hat{\alpha}\left(T C, F ; e_{S E}^{\text {ant }}(A)\right)-\frac{1}{2} e^{2} \\
= & \operatorname{Pr}(t=A \mid s) e \lambda\left[1-\frac{\alpha_{M} \rho\left[1-e_{S E}^{\text {ant }}(A)\right]}{\alpha_{M} \rho\left[1-e_{S E}^{a n t}(A)\right]+\left(1-\alpha_{M}\right)(1-\rho)}\right] \\
& +\lambda \frac{\alpha_{M} \rho\left[1-e_{S E}^{a n t}(A)\right]}{\alpha_{M} \rho\left[1-e_{S E}^{a n t}(A)\right]+\left(1-\alpha_{M}\right)(1-\rho)}-\frac{1}{2} e^{2}
\end{aligned}
$$

Maximizing (11) with respect to $e$ yields

$$
e(s)=\operatorname{Pr}(t=A \mid s) \lambda\left[1-\frac{\alpha_{M} \rho\left[1-e_{S E}^{a n t}(A)\right]}{\alpha_{M} \rho\left[1-e_{S E}^{a n t}(A)\right]+\left(1-\alpha_{M}\right)(1-\rho)}\right]
$$

In equilibrium, the anticipated effort level is equal to the optimal effort level. Thus the equilibrium effort level for an $A$ agent, denoted by $e_{S E}^{*}(A)$, is the effort level $e(A)$ which solves Equation (12) under the constraint that $e_{S E}^{a n t}(A)=e(A)$. 
Equation (12) is key in our model. The term in square brackets is the difference between the reputation from successfully performing $T C$ on the one hand, and the reputation from failing on $T C$ on the other. We refer to this term as the reputational gap. A wider gap encourages a worker to exert effort. It is easy to check that for a given effort level, an increase in $\alpha_{M}$ narrows the reputational gap. Thus, through the channel of reputation, a low expectation held by the manager encourages a worker to work hard on the challenging task. The intuition is that a worker facing a sceptical manager (low $\alpha_{M}$ ) has a lot to win in terms of reputation by successfully completing a challenging task. ${ }^{5}$ Equation (12) also shows that an $A$ worker exerts more effort than an $N A$ worker would exert if he were to opt for $T C$. To see this, note that both types of workers would gain equally much by succeeding instead of failing, but that an $A$ worker is more convinced that he is able $[\operatorname{Pr}(t=A \mid A)>\operatorname{Pr}(t=A \mid N A)]$.

The upshot is that the effect of $\alpha_{M}$ on effort is more complicated in case a reputation for being able motivates the worker than when monetary rewards motivate the worker. In both cases, the Galatea effect is present [through $\operatorname{Pr}(t=A \mid s)]$. According to this effect, an increase in $\alpha_{M}$ induces a worker to exert more effort. In the case of reputational concerns there is a counterbalancing effect through the reputational gap. As a result of the latter effect, for values of $\alpha_{M}$ close to 1, effort is close to zero. A manager who is almost sure that a worker is able attributes failure to bad luck (or an understandable lack of effort), but not to low ability. For low values of $\alpha_{M}$, the level of effort crucially depends on the probability that the worker's signal is correct, $\rho$. For high $\rho$ and low $\alpha_{M}$, the reputational gap is wide and an $A$ worker has a positive self-image in spite of the manager's negative view. In this situation, the worker exerts much effort. For low values of $\rho$ and $\alpha_{M}$, the worker has a bad self-image, inducing him to exert little effort. Figure 1 depicts the relationship between $\alpha_{M}$ and effort for various values of $\rho$ under the assumption that $s=A$ and $e(A)=e_{S E}^{*}(A)$.

Let us now examine the incentive compatibility constraints for a separating equi-

\footnotetext{
${ }^{5}$ The parameter $\rho$ has a similar effect on the reputational gap as $\alpha_{M}$. In a separating equilibrium, the higher is $\rho$, the higher is the probability that task choice contains information about the worker's ability. Performance becomes relatively less important. Therefore, the higher is $\rho$, the narrower is the reputational gap.
} 


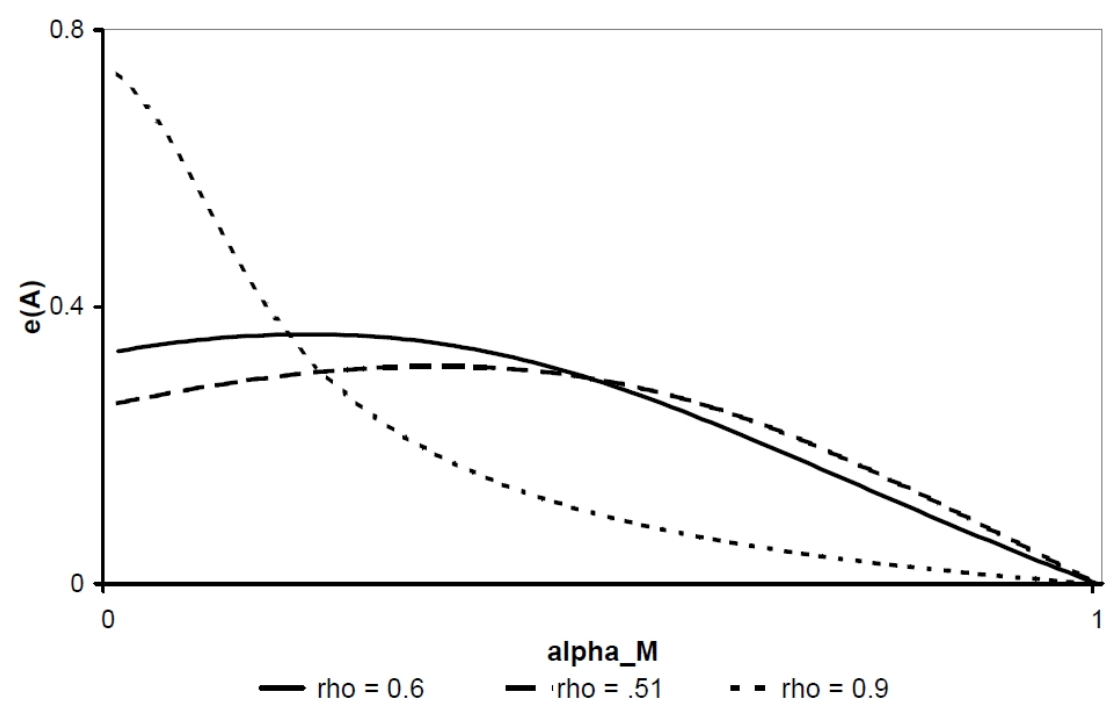

Figure 1: Optimal effort in the separating equilibrium as function of $\alpha_{M}$ when wages are fixed. Parameters: $\rho_{M}=0.5, \alpha_{W}=0.5, \lambda=1$.

librium. An $A$ worker chooses $X=T C$ rather than $X=T R$ if

$$
\begin{gathered}
\left\{\begin{array}{c}
\frac{\alpha \rho}{\alpha \rho+(1-\alpha)(1-\rho)} e_{S E}^{*}(A)+ \\
{\left[\frac{\alpha \rho\left(1-e_{S E}^{*}(A)\right)}{\alpha \rho+(1-\alpha)(1-\rho)}+\frac{(1-\alpha)(1-\rho)}{\alpha \rho+(1-\alpha)(1-\rho)}\right] \hat{\alpha}\left(T C, F, e_{S E}^{*}(A)\right)}
\end{array}\right\} \lambda \\
-\frac{1}{2}\left[e_{S E}^{*}(A)\right]^{2} \geq \hat{\alpha}(T R) \lambda
\end{gathered}
$$

An $N A$ worker chooses $X=T R$ rather than $X=T C$ if

$$
\begin{gathered}
\left\{\begin{array}{c}
\frac{\alpha(1-\rho)}{\alpha(1-\rho)+(1-\alpha) \rho} e_{S E}^{*}(N A)+ \\
{\left[\frac{\alpha(1-\rho)\left(1-e_{S E}^{*}(N A)\right)}{\alpha(1-\rho)+(1-\alpha) \rho}+\frac{(1-\alpha) \rho}{\alpha(1-\rho)+(1-\alpha) \rho}\right] \hat{\alpha}\left(T C, F, e_{S E}^{*}(A)\right)}
\end{array}\right\} \lambda \\
-\frac{1}{2}\left[e_{S E}^{*}(N A)\right]^{2} \leq \hat{\alpha}(T R) \lambda
\end{gathered}
$$

Consider the incentive compatibility constraint for an $A$ worker (13), to which we refer as ICC 1. For the moment, assume that $\alpha_{W}=\alpha_{M}$. Then, the term in parentheses equals $\frac{\alpha \rho}{\alpha \rho+(1-\alpha)(1-\rho)}$. The reason is that in equilibrium, the expected reputation equals the prior expectation. It is easy to see that the left hand-side of 
ICC 1 is always greater than zero. ${ }^{6}$ An implication is that for sufficiently high values of $\rho$, ICC 1 is always satisfied [if $\rho$ is close to $1, \hat{\alpha}(T R)=0$, see (10). For values of $\rho$ close to $\frac{1}{2}$, by contrast, ICC 1 is violated. If $\rho=\frac{1}{2}$, the expected reputation resulting from $T C$ is equal to the expected reputation from $T R$. $T C$, however, involves costly effort.

Now drop the assumption that $\alpha_{W}=\alpha_{M}$, and consider the effect of $\alpha_{M}$ on ICC 1. First, note that one of the effects of a decrease in $\alpha_{M}$ strengthens a worker's incentive to prove himself to the manager. A sceptical manager triggers a confident worker to show that he is able to perform the challenging task successfully. Choosing the routine task, by contrast, confirms the manager in her belief. The implication is that for values of $\alpha_{M}$ much lower than $\alpha_{W}$, an $A$ worker is strongly inclined to perform $T C$. Next note that if $\alpha_{M}$ is close to 1 , task performance does not have a large impact on reputations. As a result, the worker has hardly incentives to put effort in the task. The cost of performing the challenging task is therefore small. For the worker, the benefit of performing the challenging task is that this suggests that he has received a favorable signal of his ability. Thus, for both $\alpha_{M}$ being close to zero and $\alpha_{M}$ being close to 1 , an $A$ worker tends to perform the challenging task.

Does an $A$ worker also prefer $T C$ for moderate values of $\alpha_{M}$ ? We now show that for a wide range of parameters the answer to this question is in the affirmative. A clear benefit of choosing $T C$ is that if the worker successfully completes the task his ability is beyond doubt, $\hat{\alpha}(T C, S)=1$. The reputational benefit of $T R$ lies in preventing the possibility of failing on a challenging task. The expression $\left[\hat{\alpha}(T R)-\hat{\alpha}\left(T C, F, e_{S E}^{*}(A)\right)\right]$ denotes the reputational loss of failing on $T C$ relative to performing $T R$. How bad is failing on $T C$ ? In a separating equilibrium, the manager makes two inferences from failing on TC. First, the choice for $T C$ shows that the worker has received a positive signal. In isolation, choosing $T C$ thus improves the worker's reputation. Second, failing on $T C$ shows that either the worker's signal was incorrect or that effort was too low for the task. Bad performance damages the worker's reputation. So, for a worker's reputation task choice is important as well as task performance. Task choice dominates

\footnotetext{
${ }^{6}$ First note that as $e_{S E}^{*}(A)$ is optimal, we have $E\left[U\left(T C, e_{S E}^{*}(A), Z \mid A\right)\right]>E[U(T C, 0, Z \mid A)]$ $=\lambda \hat{\alpha}\left(T C, F, e_{S E}^{*}(A)\right)$. Second note that $\hat{\alpha}\left(T C, F, e_{S E}^{*}(A)\right)>0$, because we focus on the case where a worker can fail even if he is able $\left(e_{S E}^{*}(A)<1\right)$.
} 


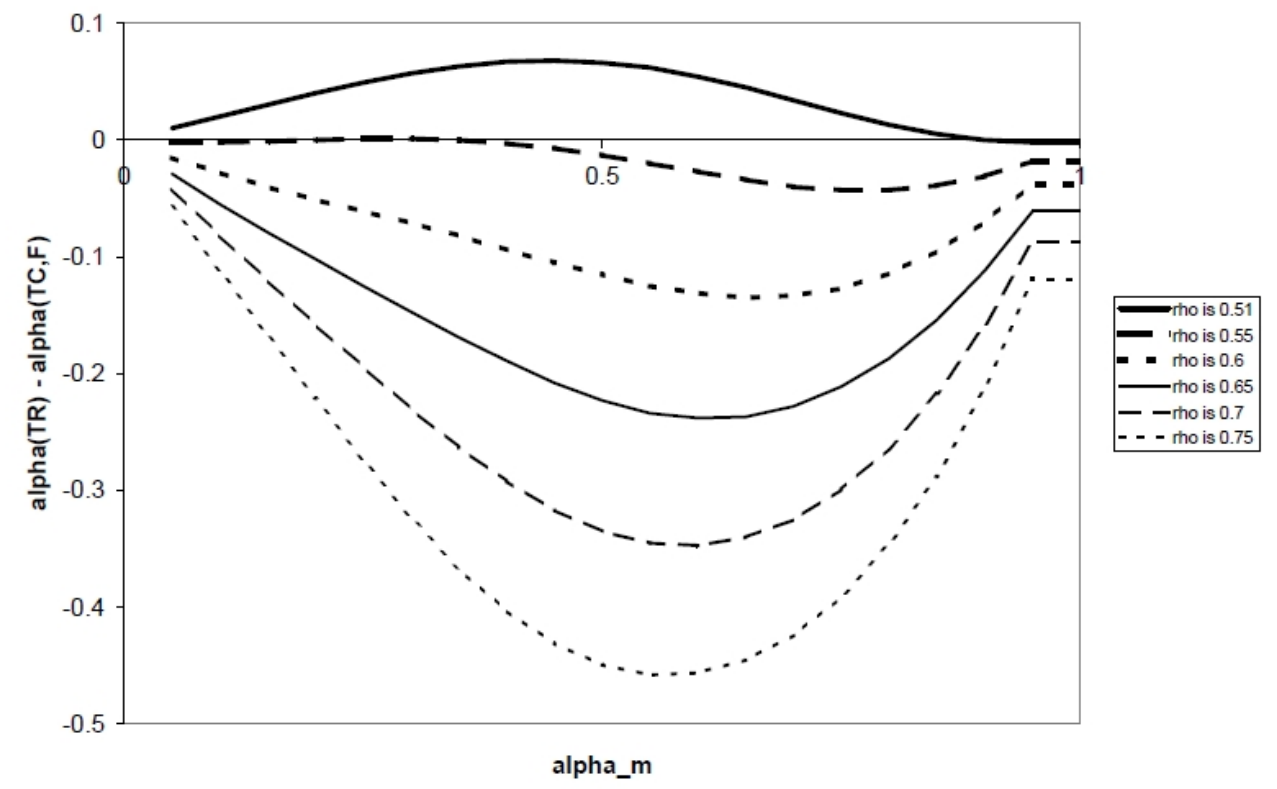

Figure 2: The potential reputational loss of doing $T C$, $\left[\hat{\alpha}(T R)-\hat{\alpha}\left(T C, F, e_{S E}^{*}(A)\right)\right]$, as a function of $\alpha_{M}$ for various levels of $\rho$. Parameters: $\rho_{M}=0.5, \alpha_{W}=0.5, \lambda=1$.

when the signal the worker receives contains much information about his ability: $\left[\hat{\alpha}(T R)-\hat{\alpha}\left(T C, F, e_{S E}^{*}(A)\right)\right]$ is decreasing in $\rho$. Figure 2 illustrates. It shows the relationship between $\left[\hat{\alpha}(T R)-\hat{\alpha}\left(T C, F, e_{S E}^{*}(A)\right)\right]$ on the one hand and $\alpha_{M}$ on the other for various values of $\rho$. Note that $\left[\hat{\alpha}(T R)-\hat{\alpha}\left(T C, F, e_{S E}^{*}(A)\right)\right]$ is negative unless $\rho$ is (very) close to 0.5. In fact our numerical analysis shows that for $\rho>0.56$, failing on $T C$ leads to a better reputation than $T R$ for all $\alpha_{M}$ Then with a view on his reputation an $A$ worker has no reason to choose $T R$. Figure 2 also illustrates that the difference between $\hat{\alpha}(T R)$ and $\hat{\alpha}\left(T C, F, e_{S E}^{*}(A)\right)$ is generally large, be it positive of negative, for moderate values of $\alpha_{M}$. The reason is that managers with strong opinions are less inclined to change their views. For instance, a manager with an extremely positive view of a worker attributes failing on $T C$ to bad luck. Managers with more moderate views are more responsive to information on task choice and task performance.

All in all ICC 1 is a mild condition. Violation requires a value of $\rho$ close to 0.5 . Moreover, violation is more likely for moderate values of $\alpha_{M}$.

We have now argued that in a separating equilibrium, an $A$ worker prefers $T C$ 


\begin{tabular}{lcccc}
\hline \hline$\alpha_{M}$ & $\rho=0.51$ & $\rho=0.52$ & $\rho=0.53$ & $\rho=0.55$ \\
\hline 0.05 & ICC2 & ICC2 & ICC2 & ICC2 \\
0.10 & ICC2 & ICC2 & ICC2 & ICC2 \\
0.15 & ICC2 & ICC2 & ICC2 & ICC2 \\
0.20 & ICC2 & ICC2 & ICC2 & ICC2 \\
0.225 & Sep.Eq. & ICC2 & ICC2 & ICC2 \\
0.25 & ICC1 & ICC2 & ICC2 & ICC2 \\
0.30 & ICC1 & Sep.Eq. & ICC2 & ICC2 \\
0.35 & ICC1 & Sep.Eq. & ICC2 & ICC2 \\
0.40 & ICC1 & ICC1 & ICC2 & ICC2 \\
0.45 & ICC1 & ICC1 & ICC2 & ICC2 \\
0.50 & ICC1 & ICC1 & ICC2 & ICC2 \\
0.55 & ICC1 & ICC1 & ICC2 & ICC2 \\
0.60 & ICC1 & ICC1 & ICC2 & ICC2 \\
0.65 & ICC1 & Sep.Eq. & ICC2 & ICC2 \\
0.70 & ICC1 & ICC2 & ICC2 & ICC2 \\
0.75 & ICC1 & ICC2 & ICC2 & ICC2 \\
0.80 & ICC1 & ICC2 & ICC2 & ICC2 \\
0.835 & Sep.Eq. & ICC2 & ICC2 & ICC2 \\
0.85 & ICC2 & ICC2 & ICC2 & ICC2 \\
0.90 & ICC2 & ICC2 & ICC2 & ICC2 \\
0.95 & ICC2 & ICC2 & ICC2 & ICC2 \\
\hline \hline
\end{tabular}

Table 1: Satisfaction of the ICCs under fixed wages

to $T R$ for a wide range of parameters. What about the incentives of $N A$ workers to choose $T C$ ? Clearly, in a separating equilibrium, an $N A$ worker has a strong incentive to choose $T C$. As shown above, for a wide range of parameters $(\rho>0.56$ be a sufficient condition), failing on $T C$ yields a better reputation than $T R$. For an $N A$ worker this means that $T C$ and no effort yields a higher payoff than $T R$. The intuition why ICC 2 is a strong condition is straightforward. In a world where workers are only concerned about their reputations for being able, we do not expect workers to choose actions that reveal that they see themselves as unable.

By emphasizing that ICC 1 is a mild condition, and ICC 2 is a strong condition we do not want to suggest that a separating equilibrium never exists. The odds for successfully completing a challenging task are higher for an $A$ worker than for an $N A$ worker. This creates room for a separating equilibrium. We do want to suggest that a separating equilibrium exists for a narrow range of parameters. Table 1 illustrates. It gives for various values of $\alpha_{M}$ and $\rho$ whether ICC 1 or ICC 2 is violated. ${ }^{7}$ We

\footnotetext{
${ }^{7}$ Note that in Table 1 we twice report a 3-decimal value of $\alpha_{M}$. We include these two values to
} 
have obtained the results for $\lambda=1, \alpha_{W}=\frac{1}{2}$ and $\rho_{M}=\frac{1}{2}$. Table 1 clearly shows that for values of $\rho$ close to 0.5 , ICC 2 is already violated. Then, an $N A$ worker chooses $T C$ rather than $T R$.

An implication of the analysis is that for a wide set of parameters a (semi) pooling equilibrium exists in which an $N A$ worker chooses the challenging task with a positive probability. ${ }^{8}$ In such equilibria, reputations depend to a large degree on task performance. In environments where workers are predominantly motivated by reputational concerns, manager can rely only to a limited extent on workers to self-select with respect to tasks.

\section{Performance Wage with Reputational Concerns}

In the previous two sections, we have discussed separately the case that workers are driven by monetary rewards, and the case that workers are driven by reputational concerns. In the present section we combine the two. We present two main results. First, we show that monetary incentives not only directly induce more effort, but that they also stimulate effort indirectly (through reputational concerns). We argue that the direct effect of monetary incentives on effort benefits the worker, but that the indirect effect harms him. Second, we show that the combination of monetary incentives and reputational concerns affects the ICCs of the separating equilibrium. We find that in an environment where in the absence of monetary incentives no separating equilibrium exists because $N A$ workers tend to choose $T C$, a reward for successfully completing TC may make a separating equilibrium possible.

How do monetary incentives affect effort choice in the presence of reputational concerns? Suppose that in our model $W_{T C}>0$ and $\lambda>0$. One can verify that in a separating equilibrium of such a model the effort level of an $A$ worker equals

$$
e_{S E}^{*}(A)=\operatorname{Pr}(t=A \mid A)\left[W_{T C}+\lambda\left[1-\hat{\alpha}\left(T C, F ; e_{S E}^{*}(A)\right)\right]\right]
$$

with $\hat{\alpha}\left(T C, F ; e_{S E}^{*}(A)\right)$ given by (9). As discussed in the previous section, failing

illustrate that there always exists a separating equilibrium in between a range of $\alpha_{M}$ where ICC 1 is violated and a range of $\alpha_{M}$ where ICC 2 is violated. The reason is that A workers are strictly more willing than NA to choose TC.

${ }^{8}$ For $\rho$ very close to $\frac{1}{2}$, and moderate values of $\alpha_{M}$, a pooling equilibrium exists in which both types of workers choose $T R$. 
on a challenging task has a large adverse effect on reputation if effort is high. The implication is a spill-over between monetary rewards and reputational rewards. An increase in $W_{T C}$ encourages an $A$ worker to exert effort. As a higher effort widens the reputational gap, an increase in $W_{T C}$ also indirectly motivates an $A$ worker. Note that the direct effect of $W_{T C}$ on effort is beneficial to the worker. He wants to exert more effort, because completing $T C$ successfully yields a higher reward. The indirect effect on effort is not beneficial for the agent. The manager anticipates the higher effort level. In equilibrium, in expected terms, the worker cannot improve his reputation. The extra effort he exerts is costly, however.

The extent to which this spillover is present crucially depends on the value of $\alpha_{M}$. For values of $\alpha_{M}$ close to zero, the spillover is negligible. In such a situation the reputational gap is close to 1 in the absence of monetary rewards. As a consequence, wages can hardly widen the gap. For values of $\alpha_{M}$ close to 1 , the spillover is also negligible because failing on a challenging task does not harm the reputation of a worker. The spillover between monetary rewards and reputation might be substantial for moderate values of $\alpha_{M}$. In such an environment, a higher equilibrium value of effort widens the gap, and task performance is important for a worker's reputation. As as result, through its effect on reputations, an increase in $W_{T C}$ induces the agent to exert more effort.

The spillover between monetary and reputational incentives influences the ICCs. In the previous section we have argued that the expression $\left[\hat{\alpha}(T R)-\hat{\alpha}\left(T C, F, e_{S E}^{*}(A)\right)\right]$ plays a crucial role for the ICCs. We have shown that in the absence of monetary rewards, failing on $T C$ sometimes leads to a slightly worse reputation than $T R$, and often leads to a better reputation than $T R$. Through its effect on effort, $W_{T C}>0$ may make $T R$ attractive from a reputational point of view. Equation (9) implies that for high equilibrium levels of effort, failing on $T C$ is detrimental for the worker's reputation.

By means of a numerical analysis, we now show that the magnitude of the effect of the spillover on the ICCs can be decisive for the existence of a separating equilibrium. ${ }^{9}$ Columns 1 and 4 in Table 2 show that in the absence of monetary incentives, for $\rho=0.53$ and $\rho=0.55$ ICC 2 is violated for all values of $\alpha_{M}$. Thus,

\footnotetext{
${ }^{9}$ We have obtained the results for $\lambda=1, \alpha_{W}=\frac{1}{2}$ and $\rho_{M}=\frac{1}{2}$.
} 


\begin{tabular}{lccccccc}
\hline \hline & \multicolumn{3}{c}{$\rho=0.53$} & & \multicolumn{3}{c}{$\rho=0.55$} \\
\cline { 2 - 3 } \cline { 6 - 7 }$\alpha_{M}$ & $W_{T C}=0$ & $W_{T C}=0.3$ & $W_{T C}=0.6$ & & $W_{T C}=0$ & $W_{T C}=0.3$ & $W_{T C}=0.6$ \\
\hline 0.05 & ICC2 & ICC2 & ICC2 & & ICC2 & ICC2 & ICC2 \\
0.10 & ICC2 & ICC2 & ICC2 & & ICC2 & ICC2 & ICC2 \\
0.15 & ICC2 & ICC2 & ICC2 & & ICC2 & ICC2 & ICC2 \\
0.20 & ICC2 & ICC2 & ICC2 & & ICC2 & ICC2 & ICC2 \\
0.225 & ICC2 & ICC2 & ICC2 & & ICC2 & ICC2 & ICC2 \\
0.25 & ICC2 & ICC2 & ICC2 & & ICC2 & ICC2 & ICC2 \\
0.30 & ICC2 & ICC2 & ICC2 & & ICC2 & ICC2 & ICC2 \\
0.35 & ICC2 & ICC2 & ICC2 & & ICC2 & ICC2 & ICC2 \\
0.40 & ICC2 & Sep.Eq. & Sep.Eq. & & ICC2 & ICC2 & ICC2 \\
0.45 & ICC2 & Sep.Eq. & Sep.Eq. & & ICC2 & ICC2 & Sep.Eq. \\
0.50 & ICC2 & Sep.Eq. & Sep.Eq. & & ICC2 & ICC2 & Sep.Eq. \\
0.55 & ICC2 & Sep.Eq. & Sep.Eq. & & ICC2 & ICC2 & Sep.Eq. \\
0.60 & ICC2 & Sep.Eq. & Sep.Eq. & & ICC2 & ICC2 & Sep.Eq. \\
0.65 & ICC2 & Sep.Eq. & ICC1 & & ICC2 & ICC2 & Sep.Eq. \\
0.70 & ICC2 & ICC2 & Sep.Eq. & & ICC2 & ICC2 & Sep.Eq. \\
0.75 & ICC2 & ICC2 & Sep.Eq. & & ICC2 & ICC2 & Sep.Eq. \\
0.80 & ICC2 & ICC2 & ICC2 & & ICC2 & ICC2 & ICC2 \\
0.835 & ICC2 & ICC2 & ICC2 & ICC2 & ICC2 & ICC2 \\
0.85 & ICC2 & ICC2 & ICC2 & ICC2 & ICC2 & ICC2 \\
0.90 & ICC2 & ICC2 & ICC2 & ICC2 & ICC2 & ICC2 \\
0.95 & ICC2 & ICC2 & ICC2 & & ICC2 & ICC2 & ICC2 \\
\hline \hline
\end{tabular}

Table 2: Satisfaction of the ICCs under performance wage and reputational concerns

in those settings, no separating equilibrium exists. $N A$ workers have too strong an incentive to choose $T C$. The second column of 2 shows that for $\rho=0.53$ and moderate values of $\alpha_{M}$, and a reward for successfully completing $T C$, a separating equilibrium does exist. So, the introduction of a monetary reward for successfully completing $T C$ may induce $N A$ workers to abstain from performing $T C$. Column 3 and 6 of Table 2 show that for higher $W_{T C}$, the scope of the parameters for which $N A$ workers choose $T R$ widens. Note that for $\rho=0.53$ and $\alpha_{M}=0.65, W_{T C}>0$ even discourages an $A$ worker to perform $T C$.

Why is the effect of $W_{T C}$ on the ICCs potentially so important? The total effect is a combination of two effects discussed earlier. First, a higher effort level affects the reputational gap between failing on $T C$ and performing $T R$. Figure 3 shows that for moderate values of $\alpha_{M}$, a higher value of $W_{T C}$ increases $\left[\hat{\alpha}(T R)-\hat{\alpha}\left(T C, F, e_{S E}^{*}(A)\right)\right]$. Again, the reason is that for high levels of effort, failing on $T C$ severely damages the worker's reputation. Second, effort directed to 


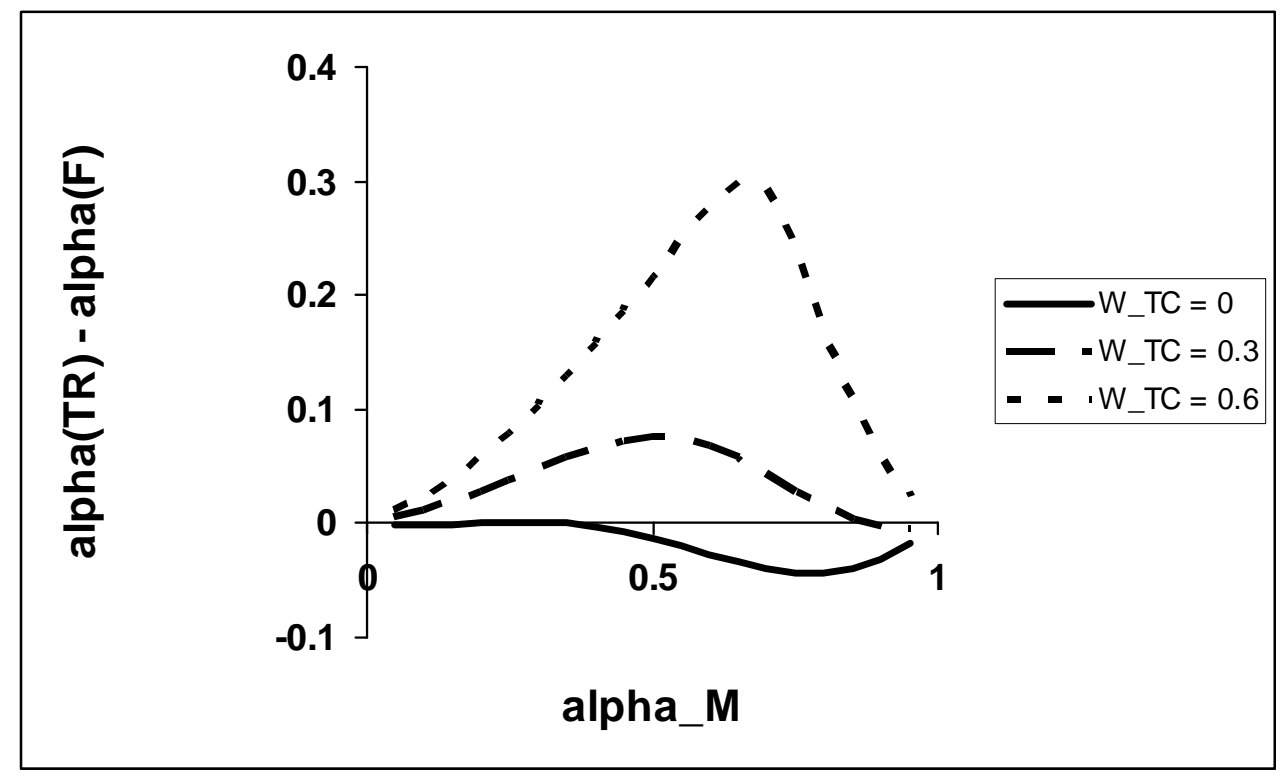

Figure 3: The potential reputational loss of doing $T C$, $\left[\hat{\alpha}(T R)-\hat{\alpha}\left(T C, F, e_{S E}^{*}(A)\right)\right]$, as a function of $\alpha_{M}$ for various levels of $W_{T C}$. Parameters: $\rho=0.55, \rho_{M}=0.5, \alpha_{W}=0.5, \lambda=1$.

improving one's reputation is a waste. Effort can affect the probability of completing a task successfully, but, in our model, does not affect expected reputations. By choosing $T R$, the worker can escape from this wasteful cost of effort.

All in all, our numerical analysis shows that in a world where workers care about money and are concerned about their reputation for being able, a monetary reward for successfully completing a task can induce workers to shy away from this task. If one interprets $W_{T C}$ as the price for performing $T R$, the routine task bears resemblance of the famous Giffen good. A cut in the price of $T R$ leads to less consumption.

\section{Conclusions}

This paper has tried to contribute to the literature on how personal characteristics of managers influence employees' behavior and companies' performance. Our focus has been on a manager's view of worker's abilities. We have derived two sets of results. First, in a model where workers are uncertain about their abilities, we have shown that in an environment where workers are driven by monetary rewards a positive 
view held by the manager encourages workers to exert effort. In environments where workers are concerned about their reputation for being able, the effect of a manager's view is more complicated. On the one hand a negative view may challenge workers to prove themselves. On the other hand, a positive view may improve workers' self-image.

The second set of results pertains to the question whether a manager should assign tasks to workers or let workers themselves choose tasks. The idea is that it might be in a manager's interest that workers with a positive self-image choose more challenging tasks, while workers with a negative self-image choose more routine tasks. We have found that when agents are primarily concerned about their reputation, the scope for self-selection is very limited. The reason is that in a world where workers may choose the task they want to perform, a choice for a routine task is a clear signal of a negative self-view. This damages a worker's reputation. A somewhat surprising result is that increasing the reward for successfully completing the challenging task may encourage workers to choose more routine tasks. The reason for this result is a spillover between monetary incentives and reputational concerns on effort. The direct effect of a higher reward is more effort. More effort, in turn, leads to a poor reputation in case of failure. Workers can avoid wasteful effort by choosing the routine task.

As usual, the results of our paper are derived from a model that is based on many assumptions. Some of them are restrictive, but innocuous. For example, our assumption that less able workers can never complete a challenging task could be replaced by the assumption that, ceteris paribus, a less able worker completes a challenging task with a lower probability than a more able worker. The math would be slightly more complicated, but the results would not be qualitatively different. In the same realm, a less innocuous assumption is that successfully completing a challenging task is a clear signal of being able, while the routine task functions as a safe haven. One could imagine situations where failing on the routine task is a clear signal of being unable. In such an environment, workers are more likely to perform the challenging task for two reasons. First, in such a model the routine task would also involve effort. Second, the routine task would no longer play the role as a safe haven.

An uncommon assumption underlying our model is that the worker infers infor- 
mation about his ability from the manager's attitude, while in the model there is no reason why the manager's attitude contains information. Our model is not fully specified in the sense that the weight the worker attributes to the probability that the manager's attitude towards workers $\left(\rho_{M}\right)$ is correct is exogenous. One advantage of this way of modelling is that it is flexible. For instance, if one believes that the manager's attitude does not contain information, one simply sets $\rho_{M}$ to zero. In that case, the well-known Galatea effect will disappear. However, the more surprising result that a negative attitude of the manager may encourage workers to exert effort would survive. One can also easily show that our result that an increase in the reward for completing a challenging task may discourage workers to perform challenging tasks does not hinge on any information asymmetry between the manager and worker. It does require that both the worker and the manager are uncertain about the worker's ability.

Finally, we have assumed an environment in which effort and ability are complements. In general, our results do not carry over to an environment where effort and ability are substitutes.

\section{References}

Ackerman, P.L., M.E. Beier and K.R. Bowen (2002), "What we really know about our abilities and our knowledge", Personality and Individual Differences, 33, 587-605.

Bandura, A. (1986), Social Foundations of Thought and Action: A social cognitive theory, Englewood Cliffs, NJ: Prentice Hall.

Banerjee, A. and R. Somanathan (2001), "A Simple Model of Voice", Quarterly Journal of Economics, 116(1), 189-227.

Baumeister, R.F. (1998). "The self", in: Gilbert, D.T., S.T. Fiske, and G. Lindzey (eds), The Handbook of Social Psychology, New York: McGraw Hill, 680740.

Bénabou, R. and J. Tirole (2003), "Intrinsic and Extrinsic Motivation", Review of Economic Studies, 70, 489-520.

Bénabou, R. and J. Tirole (2006), "Incentives and Prosocial Behavior", American Economic Review, 96(5), pages 1652-1678.

Bezuijen, X.M., P.T. van den Berg, K. van Dam and H. Thierry (2009), "Pyg- 
malion and Employee Learning: The role of leader behaviors", Journal of Management, 35(5), 1248-1267.

Che, Y,-K. and N. Kartik (2008), "Opinions as Incentives", Journal of Political Economy, 117(5), 815-860.

Dixit, A.K. and J.W. Weibull (2007), "Political Polarization", Proceedings of the National Academy of Sciences, 104, 7351-7356.

Eden, D. (1990), "Pygmalion without Interpersonal Contrast Effects: Whole groups gain from raising manager expectations", Journal of Applied Psychology, 75(4), 394-398.

Eden, D. (1992), "Leadership and Expectations: Pygmalion effects and other self-fulfilling prophecies in organizations", Leadership Quarterly, 3(4), 271-305.

Eden, D. and J. Kinnar (1991), "Modeling Galatea: Boosting self-efficacy to increase volunteering", Journal of Applied Psychology, 76(6), 770-780.

Ehrlinger J. and D. Dunning (2003), "How Chronic Self-Views Influence (and Mislead) Estimates of Performance", Journal of Personality and Social Psychology, $84,5-17$.

Hermalin, B.E. (1998), "Toward an Economic Theory of Leadership: Leading by example", American Economic Review, 88(5), 1188-1206.

Ishida, J. (2006), "Optimal Promotion Policies with the Looking-Glass Effect", Journal of Labor Economics, 24, 857-77.

Kierein, N.M. and M.A. Gold (2000), "Pygmalion in Work Organizations: A meta-analysis", Journal of Organizational Behavior, 21, 913-928.

Klar, Y., A. Medding, and D. Sarel (1996), "Nonunique Invulnerability: Singular versus distributional probabilities and unrealistic optimism in comparative risk judgments", Organizational Behavior and Human Decision Processes, 67(2), 229-245.

Köszegy, B. (2006), "Ego Utility, Overconfidence and Task Choice", Journal of the European Economic Association, 4(4), 673-707.

Kruger, J. (1999), "Lake Wobegon be gone! The "below-average effect" and the egocentric nature of comparative ability judgments", Journal of Personality and Social Psychology, 77(2), 221-232.

McNatt, D.B. (2000), "Ancient Pygmalion joins Contemporary Management: A meta-analysis of the result", Journal of Applied Psychology, 85(2), 314-322. 
Oz, S. and D. Eden (1994), "Restraining the Golem: Boosting performance by changing the interpretation of low scores", Journal of Applied Psychology, 79(5), 744-754.

Rotemberg, J.J. and G. Saloner (1993), "Leadership Style and Incentives", Management Science, 39(11), 1299-1318.

Sedikides, C. and M. Strube (1995), "Introduction to symposium", Personality and Social Psychology Bulletin, 21(12), 1277.

Shapiro, J.R., E.B. King and M.A. Quiñones (2007), "Expectations of Obese Trainees: How stigmatized trainee characteristics influence training effectiveness", Journal of Applied Psychology, 92(1), 239-249.

Swank, O.H. and B. Visser (2007), "Motivating Through Delegating Tasks or Giving Attention", Journal of Law, Economics and Organization, 23(3), 731-742.

Van den Steen, E. (2005), "Organizational Beliefs and Managerial Vision", Journal of Law, Economics, and Organization, 21(1), 256-283. 